\title{
REFLEXIÓN
} sanus

\section{Derecho al acceso a la salud de los adultos mayores en México: reflexión crítica discursiva}

\begin{abstract}
RESUMEN
Introducción: Los cambios en las condiciones de salud han logrado que la esperanza de vida de las personas aumente. La salud es un derecho fundamental y universal, por lo que las personas adultas mayores deberían gozar de él sin limitaciones. Objetivo: Reflexionar sobre las condiciones de acceso a la salud para los adultos mayores desde una perspectiva de derecho universal. Desarrollo: Se trata de un estudio reflexivo a partir de la revisión de literatura y crítica de los autores. La protección a la salud como derecho se ha enmarcado en las declaraciones internacionales y los acuerdos entre países, como un compromiso sustentable de atención al envejecimiento. México posee estrategias como la Ley General de Salud, Ley de Derechos de las Personas Adultas Mayores, el Plan de Atención al Envejecimiento. Conclusiones: La salud es un derecho universal, aún se requieren esfuerzos para lograr que todo anciano mexicano goce de este derecho con plenitud y satisfacción, teniendo una vejez óptima.
\end{abstract}

Palabras clave: Salud; Derecho a la salud; Anciano; Sistemas de Salud (DeCS, BIREME).

1Doctor en Ciencias de Enfermería, Universidad de Guanajuato, México. E-mail: ferxtom@hotmail.com

2Pasante de la Licenciatura en Derecho, Universidad Galilea.

*Autor para correspondencia

Cómo citar este artículo

Guerrero-Castañeda RF, Guerrero-Castañeda DG Derecho al acceso a la salud de los adultos mayores en México: reflexión crítica discursiva. Sanus. 2018;3(7): 56-67. [Acceso____ _ ] Disponible en: mes día año

URL 


\section{INTRODUCCIÓN}

México vive una transición demográfica interesante, el aumento de la esperanza de vida y de las condiciones económicas hacen de la población adulta mayor un crecimiento notable. A nivel mundial la población adulta mayor para el año 2015 era de 901 millones, equivalente a un $12.3 \%$ de la población ${ }^{(1)}$. En México se tiene una población de adultos mayores de casi 13 millones de personas, lo que representa el $9.6 \%$ de la población total (2).

Los cambios en las condiciones de salud han logrado que la esperanza de vida de las personas aumente, este es otra determinante del aumento poblacional, actualmente una persona puede aspirar a vivir hasta 75 años, 76 las mujeres y 74 los hombres ${ }^{(3)}$. El cuestionamiento es claro y preciso: ¿Una esperanza de vida alargada condiciona una buena calidad de vida? Las discusiones pueden ser variadas, lo que sí es seguro es que el término de esperanza de vida debe analizarse desde la perspectiva de años de vida saludables (AVS), una persona puede hoy aspirar a llegar a una edad avanzada, pero cuántos de esos años de vida transcurrirán con total independencia, autonomía y salud.

La Organización Mundial de la Salud (OMS) determina que las personas adultas mayores pueden aspirar a una calidad de vida óptima en tanto se mantengan las funciones primordiales y hábitos de vida saludables, sin embargo, muchos de los que hoy son adultos mayores ya han llegado a la vejez con alguna patología crónica, grado de dependencia y/o discapacidad, por lo que es interesante la discusión continua de los factores que condicionan estas situaciones, analizar continuamente los retos a los que se enfrenta, no sólo la población mayor, sino el sistema de salud del país, los profesionales que están capacitados para atender a estos adultos mayores y además las perspectivas con las que se atenderán, pues la curación no es el fin único ni último, sino el énfasis en la promoción y prevención. El objetivo del presente artículo es reflexionar sobre las condiciones de acceso a la salud para los adultos mayores desde una perspectiva de derecho universal.

\section{DESARROLLO}

\section{Sobre las estadísticas del acceso a la salud en México}

Las estimaciones estadísticas en relación a la población adulta mayor en el país hacen ver que habitan cerca de 13 millones de personas mayores de 60 años; en datos exactos son 12, 973, 411 personas de 60 años y más, de los cuales $53.9 \%$ son mujeres y $46.1 \%$ son hombres, según el Consejo Nacional de Población (CONAPO) ${ }^{(4,5)}$. La esperanza de vida de las personas ha ganado años, para el 2018 se reporta una esperanza de vida de 75.47 años, 78.05 para las mujeres y 73.01 para los hombres, esto es resultado de la mejora en las condiciones de salud, el avance en la economía del país y el avance de la tecnología, sin embargo, si las enfermedades crónicas eran un foco rojo por su alta mortalidad, es hora de pensar si se trata de vivir más años, cuántos de esos años serán saludables.

Una tercera parte de los adultos mayores (33.8\%) aún labora, sólo el $16.2 \%$ están pensionados y jubilados. De los que laboran, lo hacen en el comercio, servicios personales, es decir, carecen de un trabajo formal que les garantice seguridad social. Sus labores se extienden de 35 a 48 horas a la semana (36.7\%), lo que sobrepasa las horas estipuladas por la ley para laborar por día ${ }^{(4)}$.

Los ingresos por programas federales (65 y más) para el 2014 era de 5.10 millones, del adulto mayor, resaltando la importancia de que sólo un $28.1 \%$ de hombres y un $8.5 \%$ de mujeres recibe una pensión por jubilación; requiriendo aumentar este tipo de programas para aquellos que no lo tienen, pues aún no se tiene un completo acceso a seguridad social que les provee atención médica(4).

En relación a ello, sólo el $30 \%$ de los adultos mayores tiene cubierta la salud con el Seguro Popular, un 16.6\% no tiene ningún tipo de derechohabiencia ${ }^{(4)}$. La Encuesta Nacional de Ingresos y Gastos en el Hogar (ENIGH) 2016 reporta que los gastos en salud por familia por trimestre ascienden a $\$ 760.00^{(6)}$. es decir aún hay un desembolso de parte de los adultos mayores para solventar gastos relacionados con la salud, considerando nuevamente la falta de recursos en relación al tipo de actividad laboral, que sólo pocos tienen garantizado como consecuencia de una jubilación.

A ello se puede añadir la cantidad de adultos mayores dependientes, casi un $50 \%$ de los adultos mayores presenta una discapacidad, lo que se equipara con países de ingresos bajos según la $\mathrm{OMS}^{(7,8)}$. Una tercera parte reporta limitación para su autocuidado y eso se eleva a un $47.6 \%$ en los mayores de 80 años. Al menos uno de cada cuatro adultos mayores requiere ayuda para estas actividades, las principales discapacidades son las funcionales, cognitivas y visuales ${ }^{(8)}$. Esto representa un foco de atención, pues denota la importancia de las condiciones propias de la vejez que deben estar enmarcadas en el acceso a la salud.

\section{La salud se enmarca como un derecho universal}

La protección a la salud como derecho se ha enmarcado en las declaraciones internacionales y los acuerdos entre países, como un compromiso sustentable de atención al envejecimiento. En 2007, la CEPAL en su segunda Conferencia regional intergubernamental sobre el envejecimiento en América Latina y el Caribe, celebrada en Brasilia del 4 al 6 de diciembre, adopta la Declaración de Brasilia. Las principales recomendaciones de la Estrategia regional de implementación para América Latina y el Caribe del Plan de Acción Internacional de Madrid 
sobre el Envejecimiento, señala en relación a la salud y bienestar que el adulto mayor tiene derecho al acceso universal de los servicios de salud integral, la promoción de conductas y ambientes saludables, la regulación de los servicios de cuidado a largo plazo, la formación de recursos humanos en geriatría y gerontología y el seguimiento del estado de salud mental de la población adulta mayor ${ }^{(9)}$.

En México, la Ley de los Derechos de las Personas Adultas Mayores del Instituto Nacional de las Personas Adultas Mayores (INAPAM) propone que el adulto mayor "tenga acceso preferente a los servicios de salud, a gozar cabalmente del derecho a su sexualidad, bienestar físico, mental y psicoemocional; recibir orientación y capacitación en materia de salud, nutrición e higiene"(10). La Ley establece cuatro ejes rectores de su política en relación a los derechos de que todo adulto mayor debe gozar, en el artículo $4^{\circ}$, emana que los principios rectores son: la autonomía y autorrealización, donde se enmarca la claridad de que todos los adultos mayores deben tener acceso a acciones que garanticen su búsqueda de independencia y su desarrollo personal, la salud es parte fundamental del adulto mayor, como lo es parte de su sentido de vida, una persona con buena salud buscará realizarse en todas las áreas de su vida, así cuando la salud merma la vida de la persona, sus condiciones en general deprimen la búsqueda de un sentido de vida satisfactorio.

La participación como principio busca que el adulto mayor goce de tener un envejecimiento activo conceptualizado por la OMS como "el proceso de optimización de las oportunidades de salud, participación y seguridad con el fin de mejorar la calidad de vida de las personas a medida que envejecen"(11). La participación es un ejemplo del fomento de un envejecimiento activo, busca que las personas adultas mayores sean partícipes de todos los sectores en donde viven, que sean aún capaces de tomar decisiones y sean presenciados en sus ambientes familiares y comunitarios.

La equidad se enmarca como el trato justo en condiciones de acceso a la salud, se busca el bienestar de los adultos mayores de acuerdo a sus condiciones de salud y relevancia de sus patologías predominantes, siempre en favor de su bienestar, apelando al trato digno libre de discriminación, misma que puede ser por el mismo hecho de ser adulto mayor.

La corresponsabilidad, debe ser un principio que condicione una responsabilidad compartida por las instituciones que brinden atención a la salud, el reto es poder organizar este interés para que sea coherente y compartido tanto para instituciones de salud públicas y privadas, así como aquellas de asistencia social que tienen la responsabilidad de encaminar la atención al adulto mayor.

El principio de atención preferente señala que el adulto mayor tiene derecho a ser parte de programas para la atención de acuerdo a sus condiciones, incluyendo todas instituciones de salud.

El artículo $5^{\circ}$ señala los derechos de las personas adultas mayores, el tercer derecho corresponde a salud, alimentación y familia, considerando la salud como un derecho con acceso preferente de conformidad con el artículo $4^{\circ}$ constitucional, así como a la orientación en materia de $\operatorname{salud}^{(10)}$.

El artículo 18 de la misma Ley señala las responsabilidades de las instituciones públicas del Sector Salud, enunciando garantizar a los adultos mayores: derecho a la prestación de servicios públicos de calidad en atención médica, atención a programas de detección oportunay tratamiento temprano de enfermedades crónicas, personas con discapacidad; acceso a atención médica a las personas adultas mayores en clínicas y hospitales, establecimiento de áreas geriátricas en unidades médicas de segundo y tercer nivel públicas y privadas ${ }^{(10)}$. Las especialidades encargadas de la atención de la salud de las personas mayores son la Geriatría y Gerontología; tener una cartilla de salud con sus datos personales; coordinación interinstitucional para medicación, educación para la salud, apoyo a unidades médicas y civiles en la atención a la salud física y/o mental y colaboración universitaria para prestar servicios en diversas áreas, la gestión y apoyo a adultos mayores vulnerables.

La ley hace énfasis en el cuidado al adulto mayor sea en casa por la familia o por las instituciones; señalando que se requiere acceso a la salud al menos una vez al año para examen médico, mantenimiento de salud y tratamiento necesario, gozar de confidencialidad y tomar decisiones ${ }^{(10)}$.

La Ley General de Salud publicada en México en 1986 y reformada en 2014, señala desde su artículo primero el derecho a la protección a la salud del que gozan todos los ciudadanos mexicanos, enmarcado en el artículo $4^{\circ}$ de la Constitución Política de los Estados Unidos Mexicanos, bajo bases y modalidades de acceso a los servicios de salud ${ }^{(12)}$.

La protección a la salud busca el bienestar físico y mental, principios claves de la salud en el marco conceptual de la OMS, se busca además mejorar y prolongar la calidad de la vida, proteger las condiciones de salud, así como gozar de los beneficios de salud y asistencia social.

La unión de las fuerzas institucionales es una pieza clave para la atención al envejecimiento, desde la promoción y educación para un envejecimiento sano hasta la curación, prevención y promoción en aquella vejez que curse con patologías que atañen todas las esferas del ser humano. El Sistema Nacional de Salud de México tiene modalidades de atención médica, en el artículo 34 de la Ley General de Salud se encuadran los servicios públicos a la población en general, en este rubro, se adjunta el Seguro Popular, mismo que presta atención a los afiliados al Sistema de Protección Social en Salud, la cobertura de los servicios para el adulto mayor según el catálogo básico es: vacuna 
antineumocócica para adulto mayor, vacuna antiinfluenza para el adulto mayor, acciones preventivas para el adulto mayor de 60 años en adelante y diagnóstico y tratamiento de la neumonía.

Los servicios a derechohabientes de instituciones públicas de seguridad social, son los que otorgan atención a los adultos mayores afiliados y beneficiados en instituciones públicas gubernamentales, gozan además de la atención en salud médica, la asistencia social que prestan el Instituto Mexicano del Seguro Social (IMSS), el Instituto de Seguridad y Servicios Sociales para los Trabajadores del Estado (ISSSTE), Secretaría de la Defensa Nacional (SEDENA) y Petróleos Mexicanos (PEMEX). Los servicios sociales y privados, son aquellos que se contraten en cualquier modalidad, pueden corresponder tanto a la asistencia médica, así como a la social.

Cada sexenio se lanza un Programa de Acción Específico de Envejecimiento, aún vigente el 2013-2018, mismo que encuadra acciones específicas que garanticen una atención al envejecimiento. El mismo programa señala que la atención geriátrica, se basa básicamente en atender las principales causas de mortalidad de la vejez: diabetes tipo 2, enfermedades isquémicas del corazón, tumores malignos, enfermedad cerebrovascular, enfermedad hipertensiva, cirrosis hepática, enfermedad obstructiva crónica, infecciones de vías respiratorias agudas bajas, nefritis y nefrosis y accidentes ${ }^{(13)}$.

El programa hace especial énfasis en fomentar la investigación en relación a la vejez, que se implementen modelos geronto-geriátricos en el primer nivel de atención, que los sistemas referencia y contrarreferencia sean verdaderamente coordinados, así como fomentar en toda la población una cultura de envejecimiento consciente de los cambios y de las necesidades que se presentan en esta población. Es de vital importancia la capacitación al equipo de salud, es necesario saber por lo menos los cambios fisiológicos, psicológicos y sociales que se experimentan durante el proceso de envejecimiento, para poder integrar una valoración geriátrica integral que permita prevenir las deficiencias y permita revisar los recursos de los que se dispone ${ }^{(13)}$.

Si bien es cierto que las políticas de salud están vigentes, son inclusivas y coadyuvan la fuerza interinstitucional, también es cierto que las necesidades reales y las condiciones de vida de los adultos mayores en la realidad señalan el gran trabajo que se tiene que hacer para hacer totalmente visibles y palpables las políticas de atención al envejecimiento en relación a la salud.

\section{Perspectivas y retos del acceso a la salud para los adultos mayores en México}

Se ha hablado de una política incluyente, sin embargo, la cultura de integración del adulto mayor a todos los ambientes, incluida la salud no está exenta de vivir aún fenómenos de rechazo, la Encuesta Nacional sobre
Discriminación (ENADIS) 2017, señala que la mayoría de las personas se desespera con los adultos mayores (82\%). La misma encuesta señala que las principales problemáticas del adulto mayor en México son la pensión insuficiente para cubrir necesidades básicas (28.5\%), falta de oportunidades para trabajo (25.5\%) y ausencia de pensión o jubilación (21.9\%); además que el $37.0 \%$ de la población mayor recibe dinero de sus hijas e hijos para sostenerse económicamente, y el $11.0 \%$ depende únicamente de este ingreso. El análisis por sexo de esta población mayor representa el $15.1 \%$ de las mujeres y, el $6.4 \%$ de los hombres de 60 años y más ${ }^{(14)}$.

¿Por qué es necesario citar estos datos? Porque la cultura de envejecimiento aún precisa ser vinculada a toda la población desde las edades tempranas y ésta abarca la salud, una carencia del adulto mayor en México es la pensión para satisfacer necesidades básicas como la salud.

Porque el adulto mayor depende de los hijos para sostenerse, por lo tanto la atención en salud es parte fundamental del núcleo familiar, si los hijos son derechohabientes de alguna institución, el adulto mayor tendrá la plena satisfacción de la atención médica, caso contrario, si no lo son, estará recibiendo atención del seguro popular, esto hace alusión al modelo de dinámica familiar tradicional del hombre como proveedor de la familia, de tal manera que casi el $60 \%$ de las adultas mayores carece de pensión por parte del esposo, el mismo porcentaje tiene pensión universal(15).

La falta de oportunidades laborales desvirtúa también la salud, el adulto mayor que aún requiere trabajo lo hace de manera informal, por tal razón no dispone de seguridad social que le garantice atención en salud, tendrá de igual manera que ser beneficiario del seguro popular. Si este es el acceso a la salud universal resuelto por la política mexicana, entonces la necesidad de reformar continuamente y actualizar el catálogo de gastos del seguro popular debe ser una prioridad.

$\mathrm{Si}$ es un derecho y es un aporte gubernamental que se ha logrado en la historia de la política de salud, se debe fortalecer en atención a los problemas reales y necesarios de los adultos mayores; si bien es cierto que el seguro popular se visualizaría como una oportunidad en la reducción de los gastos del adulto mayor; se ha logrado aumentar su cobertura, sin embargo, el derecho a la salud como universal y estipulado en la Constitución aún tiene brechas importantes, en relación a la vejez el alcance sigue siendo limitado y está restringido como se pudo observar en su cobertura(16,17), la demanda de su cobertura a toda la población mayor requiere mejorar la infraestructura y actualizar la afiliación, así mismo la demanda de los padecimientos de la vejez requiere mayores esfuerzos.

Se sigue mostrando un gasto de bolsillo para los usuarios en un $95 \%$, la población, en un estudio de investigación, que más usa el Seguro Popular son las mujeres, no jefas de 
familia, del área rural y con un nivel educativo elemental, recibiendo además el apoyo de pensión universal para adultos mayores (PUAM) de entrega bimensual; las enfermedades de uso de servicios de salud más comunes son hipertensión más otras enfermedades y diabetes e hipertensión combinada con otra enfermedad; solo el $0,2 \%$ no padecían enfermedad. Se reporta que los gastos ascienden a cerca de $\$ 1,300.00$, en mayor cantidad en medicamentos que están en el catálogo, medicamentos no cubiertos, pago de honorarios médicos privados y exámenes de laboratorio(16).

Las brechas de desigualdad siguen siendo una realidad en el país, las condiciones de pobreza de los adultos mayores, consecuencia de la falta de seguridad social y de la oportunidad laboral reflejan pobreza en la vejez en un $74.7 \%$ de la cual el $23.7 \%$ cae en indigencia ${ }^{(15)}$; aquí se suma la asistencia social como parte de la atención a la salud.

El concepto de vulnerable en la población adulta mayor concierne a la disminución de los recursos activos, tanto personales como familiares y comunitarios, que genera exclusión social y pobreza, además de tener un impacto en las necesidades indispensables, como lo es la salud, pues se torna no un derecho sino un privilegio para los que tienen recursos para su acceso.

El deterioro de la salud de los mayores tiene diversas condicionantes que van desde las características propias de la persona hasta situaciones de índole contextual ${ }^{(4)}$, sin embargo, lo que es seguro es que ese deterioro del estado de salud tiene una fuerte influencia sobre la morbilidad en la vejez, por lo que la búsqueda de los servicios de salud será un continuo en diferentes niveles de atención de salud, así como en el requerir servicios acordes a la situación epidemiológica predominante, donde se puede analizar la situación de género, edad, apoyos estatales y el soporte familiar para tener acceso a los servicios, así como las necesidades de cuidado que el adulto mayor pueda requerir.

Es necesario fortalecer la atención primaria de la salud como un recurso que atienda la vejez en todas sus esferas, la reforma del primer nivel desde las instituciones educativas debe ir más allá de las metas establecidas y buscar una reforma verdaderamente incluyente práctica, es necesario antes que todo, que el ahorro en cuentas individuales sea una realidad,

Se requiere una mejora de los servicios de salud, sin brechas, que sean los servicios los que lleguen a las comunidades, un fortalecimiento de la atención primaria con énfasis en una cultura de envejecimiento saludable que permeé en todo profesional de la salud, es necesario también vincular a asociaciones civiles y a la población en general a través de redes de apoyo social.

\section{CONCLUSIONES}

Existe en el país una política de acceso a la salud, pues es un derecho humano universal del cual los adultos mayores también gozan, sin embargo, una gran cantidad de adultos mayores vive en situación de vulnerabilidad en este derecho, por lo que de la política a la práctica seguramente hay un camino largo para reducir las brechas de este sector poblacional.

Es necesario que la política de acceso a la salud sea verdaderamente universal, que haya una vinculación institucional que responda a las necesidades de salud de los adultos mayores considerando la transición epidemiológica propia del país y las enfermedades crónicas más frecuentes en esta población; quizá una reestructuración de todo el sistema de salud sería un tema a analizar dadas las características de la salud mexicana.

Para poder lograr un acceso a la salud sin brechas de desigualdad, es necesario comprender en su totalidad las características de los adultos mayores mexicanos, sus diferencias según la zona del país, las condiciones de las política estatales que pueden no alcanzar a cubrir la expectativa de un plan de acción nacional, además analizar las condiciones de la vejez por género, proceso de salud-enfermedad, desigualdad económica y laboral, además del apoyo de otro sectores. La inclusión de la familia como protectora de la salud de los adultos mayores resulta crucial, sin embargo, esa cultura de envejecimiento debe tener responsabilidades bien definidas, de manera que la salud de los mayores no sea vista como una carga, por el contrario, que se busque la integración colectiva bajo una visión de fortalecimiento social y de la salud en la población general.

\section{REFERENCIAS BIBLIOGRÁFICAS}

1. Helpage. Global AgeWatch Index 2015: Insight report [Internet]. London; 2015 [citado el 13 de enero de 2017]. Disponible en: http://www.helpage.org/globalagewatch/reports/global-agewatch-index-2015-insightreport-summary-and-methodology/

2. HelpAge International. Global Age Watch Index 2015: Report Card México [Internet]. AgeWatch report card México. 2015 [citado el 6 de junio de 2016]. Disponible en: http://www.helpage.org/global-agewatch/populationageing-data/country-ageing-data/?country=Mexico

3. Instituto Nacional de Estadística y Geografía (INEGI). Población. Esperanza de vida [Internet]. INEGI. 2017 [citado el 11 de septiembre de 2017]. RDisponible en: http://cuentame.inegi.org.mx/poblacion/esperanza. aspx?tema $=$ P.

4. Instituto Nacional de las Mujeres. Situación de las 
personas adultas mayores en México [Internet]. México; 2015 [citado el 4 de noviembre de 2016]. Disponible en: $\quad$ http://cedoc.inmujeres.gob.mx/documentos_ download/101243_1.pdf

5. CONAPO. Proyecciones de la Población 20102050 [Internet]. Proyecciones de la Población 20102050. México; 2018 [citado el 22 de agosto de 2018]. Disponible en: http://www.conapo.gob.mx/es/CONAPO/ Proyecciones

6. INEGI. Encuesta Nacional de Ingresos y Gastos de los Hogares 2016 [Internet]. Encuesta Nacional de Ingresos y Gastos de los Hogares 2016. 2016 [citado el 21 de agosto de 2018]. Disponible en: http://www.beta.inegi.org.mx/ proyectos/enchogares/regulares/enigh/nc/2016/

7. INEGI. Encuesta Intercensal 2015 [Internet]. Encuesta Intercensal 2015. 2015 [citado el 11 de septiembre de 2017]. Disponible en: http://www.beta.inegi.org.mx/ proyectos/enchogares/especiales/intercensal/

8. INSP. Encuesta Nacional de Salud y Nutrición 2012. Discapacidad y dependencia en adultos mayores mexicanos: un curso sano para una vejez plena [Internet]. México; 2012 [citado el 22 de agosto de 2018]. Disponible en: http://ensanut.insp.mx

9. Stang F, Stenger J, Tapia Diseño P, Leyton AV. Los derechos de las personas mayores [Internet]. Santiago de Chile: Naciones Unidas; 2011 [citado el 21 de agosto de 2018]. Disponible en: http://www.cepal.org/celade/ envejecimiento

10. INAPAM. Ley de los Derechos de las Personas Adultas Mayores [Internet]. Ley de los Derechos de las Personas Adultas Mayores. 2002 [citado el 21 de agosto de 2018]. Disponible en: http://www.salud.gob.mx/unidades/cdi/ nom/compi/ldpam.html

11. Organización Mundial de la Salud. Informe Mundial sobre el envejecimiento y la salud. Suiza: OMS; 2015.

12. Secretaría de Salud. Ley General de Salud [Internet]. México; 2014 [citado el 21 de agosto de 2018]. Disponible en: http://www.salud.gob.mx/cnts/pdfs/LEY_GENERAL_ DE_SALUD.pdf

13. Secretaría de Salud. Atención del Envejecimiento 20132018 Programa Sectorial de Salud [Internet]. México, DF: Secretaría de Salud México; 2014. 104 p. Disponible en: http://www.cenaprece.salud.gob.mx/descargas/pdf/PAE_ AtencionEnvejecimiento2013_2018.pdf

14. CONAPRED. Comunidad de Prensa Encuesta Nacional sobre Discriminación (ENADIS) 22017 [Internet]. México;
2018 [citado el 21 de agosto de 2018]. Disponible en: http://www.conapred.org.mx

15. Damián A. Seguridad social, pensiones y pobreza de los adultos mayores en México. Acta Sociológica [Internet]. 2016 [citado el 9 de mayo de 2017];70:151-72. Disponible en: http://www.sciencedirect.com/science/ article/pii/S0186602817300075

16. Pavón-León P, Reyes-Morales H, Martínez AJ, MéndezMaín SM, Gogeascoechea-Trejo M del C, Blázquez-Morales MSL. Gasto de bolsillo en adultos mayores afiliados a un seguro público de salud en México. Gac Sanit [Internet]. 2017 [citado el 21 de agosto de 2018];31(4):286-91. Disponible en: https://www.sciencedirect.com/science/ article/pii/S0213911117300444

17. Juárez-Ramírez $C$, Márquez-Serrano $M$, Salgado de Snyder N, Pelcastre-Villafuerte BE, Ruelas-González MG, Reyes-Morales H. La desigualdad en salud de grupos vulnerables de México: adultos mayores, indígenas y migrantes. Rev Panam Salud Pública [Internet]. 2014 [citado el 21 de agosto de 2018];35:284-90. Disponible en: https://www.scielosp.org/article/rpsp/2014.v35n4/284290/ 